\title{
Comparación del Consumo de Drogas en una Población Escolar de Género Masculino Utilizando la Metodología de Pares y el Cuestionario Auto Registrado Estándar
}

\section{Drug Consumption in The Masculine School Population Using The Peer Methodology and The Standard Self Assessment Questionnaire}

\author{
Jorge Rodríguez T. \\ Universidad del Desarrollo, Chile \\ Daniela Dalbosco R. \\ Consultorio Atención Primaria, Chile \\ María Javiera Lee A. \\ Hospital Félix Bulnes, Chile
}

\author{
Macarena Valdés C. \\ Universidad de Chile \\ Marianela Hoffman S. \\ Universidad del Desarrollo, Chile \\ Rafael Ramírez F. \\ Universidad de Chile \\ $\&$ \\ Matías Pruzzo G. \\ Universidad de Chile
}

\author{
Ana María Fernández \\ Universidad de Santiago de Chile \\ María Fernanda Flores O. \\ Universidad del Desarrollo, Chile \\ Enrica Ramírez P. \\ Universidad de Chile
}

(Rec: 10 de Agosto de 2010 / Acep: 25 de Agosto de 2011)

\section{Resumen}

El objetivo es comparar el consumo de drogas a través de dos metodologías, la metodología de pares (MP) y la metodología del cuestionario auto registrado (CAR). La población está compuesta por escolares de género masculino, que cursan entre $8^{\circ}$ básico y $4^{\circ}$ medio. La muestra alcanzó a 301 escolares. Los resultados obtenidos son, una tendencia a mayor consumo general con la MP que con el CAR; igualmente, en el último mes el consumo muestra una tendencia al aumento con la MP en comparación con el CAR. En ambos períodos no hubo significación estadística. Por otro lado, el consumo de drogas ilícitas en los últimos 12 meses, fue el doble con la MP. En cuanto al consumo de los últimos 30 días, también se duplica al indagar con la MP. La declaración del consumo de drogas ilícitas es significativamente mayor con la MP que con el CAR, en ambos períodos estudiados. El consumo de marihuana, en ambos períodos, es el de mayor consumo entre las drogas ilícitas.

Todas las diferencias alcanzaron significación y por lo tanto, la hipótesis que indica utilizando la MP se obtiene mayor declaración del consumo de drogas ilícitas se ratifica.

Palabras claves: Metodología de pares, consumo de drogas, escolares, hombres.

\begin{abstract}
The objective of this study is to compare the declared licit and illicit drug consumption, between paired methodology (MP) and self assessed questionnaire (CAR). We suggest that understatement of illicit drug use will be minimized with the use of peer methodology, and that licit drug understatement will not be affected using peer methodology. The population of this study is composed by male school students ranging between middle and high School. The sample reached to 301 students. The results obtained regarding illicit drug consumption in the year, tend to be higher using MP compared with the CAR. Similarly, last month consumption reached a higher prevalence using MP than using CAR. There are no significant differences in both samples, during both periods. Nevertheless, in the last 12 months, illicit drug consumption doubled the CAR report in comparison to MP. Regarding drug consumption in the last 30 days, the results also showed that CAR underestimated half of the rate stated using MP. Illicit drug consumption is significantly higher using MP, than CAR, in both periods that were studied. Illicit consumption rates of in the past year and in the last month are significantly higher using MP, particularly with Marihuana. There are no statistical differences in the same periods, regarding licit drug consumption rate, as alcohol and tobacco.
\end{abstract}

Key words: Drug consumption, peer methodology, school population, men.

\footnotetext{
Correspondencia: Jorge Rodríguez T., Centro de Estudios Evolutivos e Intervención en el Niño (CEEIN), (Facultad de Psicología), Universidad del Desa-
} rrollo, dirección, Santiago, Chile. E-mail: jrodrigu80@hotmail.com 


\section{Introducción}

El consumo de drogas es un problema de salud generalizado en la población; ya en el año 2002 se planteó que el $8,9 \%$ de la carga de morbilidad total está relacionada con el consumo de sustancias psicoactivas. El tabaco representa un $46,1 \%$, alcohol $44,9 \%$ y las drogas ilícitas un $9 \%$. El consumo de drogas ilícitas es de $2,7 \%$ en la población general y de 3,9\% en la población de más de 15 años (Organización Mundial de la Salud, 2004).

Se estima una prevalencia mundial de $4,2 \%$ para cualquier tipo de droga ilícita en la población de 15 años y más. La amplia disponibilidad en la región (se estima que el $95 \%$ de la producción mundial se da en la región andina y el $70 \%$ del consumo ocurre en América), hace cada vez más favorable el inicio en el consumo como el consumo en sí. En Argentina es de 2,3\%, Chile 2,1\%, Colombia 1,2\%, Perú $1 \%$ y Estados Unidos un 3\% (United Nations Office on Drug Control and Crime Prevention, 2002).

Todos los trabajos científicos desarrollados muestran que la drogadicción es un problema latente y en aumento en el mundo. Por ejemplo, en un estudio reciente llevado a cabo con adolescentes en Bangkok, se encontró que 5,4\% consume cigarrillos, 37,3\% consume alcohol y 37,8\% consume drogas ilícitas. La comparación de la prevalencia del consumo de drogas en estudiantes universitarios de medicina en países desarrollados muestra una baja del consumo de cigarrillos (del 28,8\% al 9,2\%), en contraste a una fuerte alza del consumo de alcohol. Asimismo, investigaciones recientes en universitarios de Sao Paulo, muestran que el consumo de drogas lícitas ha aumentado en mujeres, mientras que el consumo excesivo tiende a ser mayor en el sexo masculino (86,5\% para el tabaco y $65 \%$ para el alcohol). Por otro lado, se ha encontrado una fuerte relación explicativa, en un plazo de cinco años, entre el consumo de drogas, la violencia y victimización. Dentro del consumo de drogas en nuestro país, el alcoholismo es una de las mayores preocupaciones, que está atacando cada vez con superior fuerza a la población de jóvenes (Boland et al., 2002; Pillon, O'Brien \& Piedra, 2005; Ruanghanchanasetr, Plitponkarnpim, Hetrakul \& Kongsakon, 2005; Weiner, Sussman, Sun \& Dent, 2005).

Según lo reportado por Villatoro-Velázquez, MedinaMora, Hernández-Valdés, FleizBautista, AmadorBuenabad et al., el 2005, el consumo de drogas entre adolescentes mexicanos de 12 a 17 años, muestra que el consumo de marihuana en varones es de un $2,5 \%$ y de cocaína de un $1 \%$. Y en cuanto al consumo actual alcanzaría un $1,1 \%$ la marihuana y $0,2 \%$ la cocaína, que serían las drogas que con mayor frecuencia siguen consumiendo los adolescentes.

También en México en el año 2000, el porcentaje de consumo de tabaco fue 50,7\%, alcohol 61,4\% y el consumo de drogas ilícitas 14,7\%. En cambio la Encuesta Nacional de
Adicciones en el 2002, encontró que en adolescentes de 12 a 17 años, el consumo de alcohol es de 25,7\%; alcanzando un 35\% en el sector urbano y $18 \%$ en el área rural. El abuso de alcohol sigue aumentando, llegando a un $23,8 \%$ en la población escolar de nivel medio y medio superior. Por otro lado, el consumo de tabaco en los hombres es $15,4 \%$, consumo menor que el alcohol, y en cuanto al consumo de drogas ilícitas, especialmente la marihuana en población escolar masculina, se observa un aumento progresivo. En estudios europeos realizados en adolescentes de 14 a 18 años, se observa un aumento importante del consumo de cocaína en los últimos 10 años, llegando a un 7,2\%, un aumento promedio de 40\% anual (Encuesta Nacional de las Adicciones, 2002; Medina-Mora, Cravioto, Villatoro, Fleiz, Galván-Castillo, Tapia-Conyer, 2003; Organización Mundial de la Salud, 2006. En Chile el Consejo Nacional para el Control de Estupefacientes (CONACE, 2006) viene realizando estudios bianuales en la población general desde 1994 y en la población escolar desde el año 1995, sin embargo cabe destacar que los resultados no han sido alentadores. En el 2005 el consumo de marihuana fue de un 15,6\% en comparación con $13,1 \%$ y $14,7 \%$ que habían sido las estimaciones de los estudios anteriores, lo que representa un aumento promedio anual del orden del 4,0\%. Este incremento se concentra en los Cuartos Medios, aumentando de un 22,5\% a un 27,5\%, lo que resulta concordante con el aumento detectado del uso de marihuana en jóvenes que se encontró en el último estudio en la población general. En cuanto a los porcentajes encontrados en relación al tabaco, estos se mantienen altos y estables en el mismo período. La prevalencia del último mes está entre un $39 \%$ y $41 \%$ en los últimos doce años y el consumo diario de tabaco está alrededor del $29 \%$. Por su parte, la prevalencia del alcohol en el último mes alcanzó un 57\%, siendo estable en diferentes edades evaluadas en hombres. Cabe destacar que el uso de alcohol ha aumentado significativamente en el país desde 39\% en 1994 hasta 57\% en 2006, es decir ha registrado un aumento de un $46 \%$ (CONACE, 2006). Por otro lado, las personas que declaran consumir diariamente alcohol o casi todos los días, alcanzan al 2,5\% de la población.

Los resultados de los estudios parecen preocupantes por las implicancias en trastornos mentales, más aún, si se considera que el abuso de alcohol en Chile se ha mantenido estable en el periodo estudiado, llegando a una tasa de abuso de un 13\% (DSM IV, 2005; Kohn et al., 2005).

Por otro lado, se ha observado que en todas las conductas de riesgo que tienen un castigo social y/o legal, tales como el aborto provocado, el maltrato intrafamiliar, maltrato entre pares (bullying), consumo de drogas, existe una tendencia a no declarar esa conducta. Sin embargo, existe una variedad de metodologías orientadas a ubicar poblaciones ocultas como los consumidores de drogas. Una de estas metodologías es la denominada entrevistador de acceso privilegiado (EAP), que ha sido utilizada con mucho éxito en Europa (Fountain \& Griffiths, 1999; Wiebel, 1990). 
Dentro de los grupos más afectados por la sub declaración del consumo de drogas ilícitas estarían los hombres entre 18 y 25 años, y los escolares entre $4^{\circ}$ básico y $4^{\circ}$ medio. De hecho, los niveles de consumo encontrados aplicando la metodología de pares, muestra una situación muy preocupante para el grupo de jóvenes, ya que el porcentaje se ubica por sobre los índices de consumo en los últimos 30 días, aplicando las metodologías tradicionales. Los resultados obtenidos en los últimos 12 meses en relación al consumo de drogas lícitas fue 92,3\%, encontrando 80,3\% de alcohol y consumo de tabaco 78,9\%. La tasa de consumo encontrada fue $63,4 \%$ de marihuana, $4,9 \%$ de cocaína y $2,1 \%$ de éxtasis, significativas y superiores en relación con las estimaciones encontradas con entrevistas tradicionales. Cobran aún mayor relevancia si se asocian con conductas de riesgo como la violencia (Rodríguez, Fernández \& Hernández, 2007; Rodríguez, Hernández \& Cumsille, 2005).

En estudios de poblaciones cautivas (cerradas) en relación al consumo de drogas ilícitas a través de la MP, se han encontrado niveles de consumo muy superiores a los reportados con las metodologías tradicionales (Rodríguez, J.; Hernández, E.; \& Cumsille, M.A., 2005; Rodríguez, J.; Fernández A.M.; Hernández, E. \& Ramírez, S., 2006; Jorge Rodríguez, Ana María Fernández, 2007). Considerando lo anterior, resulta necesario desarrollar metodologías que logren disminuir la sub declaración de información en temas con alta prevalencia y relevancia para la salud mental escolar y juvenil, como es el consumo de drogas.

En los trabajos publicados en relación al tema del consumo de drogas, hay una permanente preocupación sobre los indicadores que se obtienen, de hecho los porcentajes o tasas de consumo tienen una alta variabilidad, dependiendo de la institución que la realiza, lugar, momento, población a estudiar, modo de selección, instrumento utilizado y en especial la forma de recolección de los datos. Todo lo anteriormente mencionado, respalda la propuesta de la presente investigación, de evaluar una nueva forma de recolección de datos, frente a la potencial existencia de poblaciones ocultas en los trabajos de prevalencia, porcentajes, tasas de consumo de drogas, que permita a las autoridades del área de la salud tener una aproximación confiable a las magnitudes del consumo de la población escolar y de esta forma crear estrategias de intervención adecuadas.

\section{Método}

\section{Diseño}

Estudio correlacional con diseño de tipo transversal, en una muestra escolar dirigida a los establecimientos que accedieron a participar.

\section{Participantes}

La población estuvo constituida por escolares de género masculino que cursaban entre $8^{\circ}$ básico a $4^{\circ}$ medio, en colegios de la comuna de Recoleta y Huechuraba, durante el año 2006. En ellos se evaluaron los índices de consumo de drogas lícitas e ilícitas, utilizando dos métodos paralelos de recolección de datos, la metodología del cuestionario auto registrado y la metodología de pares.

El tamaño muestral calculado fue de 316 escolares, 158 con la metodología de pares y 158 con la de Cuestionario auto registrado, dentro del cual se consideró el nivel de consumo de drogas en esta población de un 5,0\% $(12,13)$, con un nivel de confianza de $95 \%$, y una diferencia máxima aceptable de los consumos de drogas entre ambas metodologías de 4,8\%. La muestra definitiva fue de 301 escolares, 152 con el Cuestionario auto registrado y de 149 escolares con la metodología de pares, La muestra final representa un 95,3\% del tamaño original, donde un 50,5\% de los alumnos evaluados fueron con el Cuestionario auto registrado. La edad promedio para ambos grupos fue de 14,5 años y desviación estándar de 1,4 años, el rango de variación estuvo entre 12 y 21 años.

\section{Procedimiento}

Se seleccionaron cuatro colegios dentro de las comunas que convinieron realizar el estudio, los que se asignaron aleatoriamente a las metodologías, cuidando que los índices de vulnerabilidad de los colegios fueran similares para garantizar homogeneidad dentro de la muestra.

En los colegios donde se aplicó la metodología de pares, se siguieron los siguientes pasos para la selección de los evaluadores:

1. Se realizaron charlas de información sobre el estudio, solicitando la participación voluntaria de los escolares, tanto para ser entrevistador como entrevistado, en todos los cursos.

2. A los alumnos reclutados voluntariamente para aplicar la entrevista se les explicó en detalle los objetivos del estudio y después se realizó una entrevista a cada uno, para posteriormente seleccionar a aquellos que cumplían ciertos requisitos, como el no tener ninguna aprensión o discriminación respecto al tema de drogadicción en general.

3. Los alumnos seleccionados para ser entrevistadores (dos de un colegio y cuatro del otro) fueron capacitados para aplicar la entrevista en forma independiente en cada colegio.

En la primera sesión de capacitación, se explicaron nuevamente los objetivos del estudio, el instrumento a usar y la forma de aplicación, enfatizando el anonimato y privacidad del procedimiento, así como también se enfatizó 
la necesidad de realizar la entrevista en lugares donde los participantes se sintieran cómodos. En la segunda y tercera sesión, los entrevistadores se aplicaron el instrumento entre ellos (role-playing).

Posteriormente se evaluó el número total de alumnos de ambos colegios. Los entrevistadores fueron supervisados por los investigadores durante todo el proceso de recolección, manteniendo una comunicación permanente y solucionando las dudas.

En los dos colegios restantes se aplicó la Metodología del Cuestionario auto registrado. Bajo el Cuestionario auto registrado, se trabajó con cuatro entrevistadores experimentados por colegio, los que eran ajenos al colegio seleccionado. Los entrevistadores en cada curso entregaron las instrucciones en voz alta, poniendo énfasis en el anonimato y confidencialidad de la información que los alumnos entregarían. Posteriormente le entregaron el instrumento a cada alumno para ser respondido y una vez finalizado el cuestionario, el alumno devuelve el instrumento al entrevistador.

\section{Variables}

Se consideraron drogas lícitas al tabaco y alcohol, y drogas ilícitas a la marihuana, cocaína, pasta base, éxtasis y otras. De esta forma, se define al fumador como aquel que consume tabaco al menos dos veces por semana; así como el consumidor de alcohol o marihuana se define por consumo de al menos una vez por semana. En el caso del resto de las drogas (cocaína, éxtasis, pasta base u otra), el criterio es que exista consumo al menos una vez al mes.

\section{Instrumento}

En la evaluación realizada con ambas metodologías se utilizó un instrumento adaptado especialmente para esta población, donde se eliminaron algunas preguntas del cuestionario original, el cual fue validado por Rodríguez \& Hernández (2005) para medir consumo de drogas en jóvenes universitarios. Posteriormente esta adaptación se aplicó a una muestra de 20 escolares para evaluar el entendimiento del lenguaje utilizado.

El instrumento obtiene información sobre el consumo de: drogas lícitas (tabaco, alcohol) y drogas ilícitas (marihuana, cocaína, pasta base, éxtasis e inhaladores). También, mide comportamientos o conductas antisociales como riñas o peleas callejeras, intentos de suicidio y creencias religiosas.

\section{Análisis de datos}

Una vez obtenida la información se ingresaron los datos al programa SPSS, versión 17.0, donde se realizó un análisis descriptivo resumiendo la información en indicadores de tendencia central y de dispersión.

Para la comparación de los promedios (frecuencia de consumo) a nivel global, con ambas metodologías, tanto para drogas lícitas como ilícitas, así como cada una de forma individual, se realizó la prueba estadística de T de Student o Mann Whitney, en función del cumplimiento de la normalidad y varianzas iguales, necesarias para realizar la prueba.

En el caso de variables de tipo cualitativo, como el consumo en drogas lícitas y drogas ilícitas, se utilizó la prueba de diferencia de proporciones $(Z)$ o la prueba exacta de Fisher. Para todos los análisis se consideró significativo un valor menor o igual a 0.05 , en las pruebas tanto unilateral como bilateral.

\section{Resultados}

En relación a los resultados obtenidos en una muestra de 301 escolares, con edades entre 12 y 21 años, el promedio encontrado fue de 14,5 años y la desviación estándar de 1,4 años. También se encontró que el 90,6\% de los niños vive con al menos alguno de los padres y el $67,9 \%$ vive con ambos. Por otro lado, el 29,6\% de los padres no tiene educación y el 25,6\% de las madres tampoco. El 7,6\% de los escolares declara que su papá tiene educación superior y el 8,0\% que sus madres también, donde alrededor del 25\% de los padres llega a la educación media. Por otra parte, se encontró que el 77,7\% de los padres y el 52\% de las madres tienen un trabajo estable, y el 2,7\% de los padres y el 16,3\% de las madres no lo tienen.

El 53,9\% de los escolares declaró que consumen alguna droga lícita en el año y el 27,6\% refieren que lo hace mensualmente. Al comparar el consumo de drogas anual entre las metodologías de pares y el cuestionario auto registrado, las tasas declaradas fueron de $58,8 \%$ y $49,0 \%$ respectivamente en el año y de $31,5 \%$ y $23,7 \%$ en relación al consumo actual, no siendo significativas las diferencias observadas. El consumo actual representa el $51 \%$ del consumo anual (ver Tabla 1).

El consumo anual de tabaco alcanzó a un 32,7\%, del cual se registró un $37,2 \%$ con la metodología de pares y $28,2 \%$ con la metodología del cuestionario auto registrado, por otro lado, el consumo actual referido fue de 18,3\%, 21,5\% y $15,1 \%$ respectivamente. En la comparación de las tasas de consumo, las diferencias no alcanzaron significación estadística (ver Tabla 2).

En la comparación del consumo de alcohol entre ambas metodologías, tampoco hubo diferencias significativas, aunque con la metodología de pares fue ligeramente superior en el consumo anual, no así en el consumo actual, como se observa en la Tabla 3.

En los consumidores anuales de tabaco, alcohol y marihuana, la edad promedio de inicio del consumo de tabaco, es significativamente menor con la metodología de pares que con la metodología del cuestionario auto registrado y la cantidad promedio de consumo de tabaco es significativamente mayor con la metodología de pares que con la metodología 
Metodología de Pares y el Cuestionario Auto Registrado Estándar

Tabla 1: Porcentaje de consumo de drogas lícitas según metodología.

\begin{tabular}{ccccccc}
\hline Período de consumo & CAR $\mathrm{n}=149$ & MP $\mathrm{n}=148$ & Total & Prueba $Z$ & Valor $p$ \\
\hline Último año & $7349,0 \%$ & $8758,8 \%$ & $16053,9 \%$ & 1,692 & $0,104 *$ \\
Últimos 30 días & $36 \quad 23,7 \%$ & $4731,5 \%$ & $83 \quad 27,6 \%$ & 1,525 & $0,156 *$ \\
\hline
\end{tabular}

$* \mathrm{p}>0.05$

$\mathrm{CAR}=$ cuestionario auto registrado, MP = metodología de pares.

Tabla 2: Porcentaje de consumo de tabaco según metodología.

\begin{tabular}{lrrccc}
\hline Período de consumo & CAR n=149 & MP $n=148$ & Total & Prueba $Z$ & Valor $p$ \\
\hline Último año & $4228,2 \%$ & $5537,2 \%$ & $9732,7 \%$ & 1,525 & $0,109 *$ \\
Últimos 30 días & $2315,1 \%$ & $3221,5 \%$ & $5518,3 \%$ & 1,424 & $0,180 *$ \\
\hline
\end{tabular}

$* \mathrm{p}>0.05$

$\mathrm{CAR}=$ cuestionario auto registrado, $\mathrm{MP}=$ metodología de pares.

Tabla 3: Porcentaje de consumo de alcohol según metodología.

\begin{tabular}{lrcccc}
\hline Período de consumo & CAR $n=152$ & MP n=149 & Total & Prueba $Z$ & Valor $p$ \\
\hline Último año & $5938,8 \%$ & $6644,3 \%$ & $12541,5 \%$ & 0,964 & $0,351^{*}$ \\
Últimos 30 días & $2617,1 \%$ & $2617,4 \%$ & $5217,3 \%$ & 0,077 & $0,937 *$ \\
\hline
\end{tabular}

$* \mathrm{p}>0.05$

$\mathrm{CAR}=$ cuestionario auto registrado, $\mathrm{MP}=$ metodología de pares.

Tabla 4: Indicadores de edad y consumo de sustancias según metodología

\begin{tabular}{lccc}
\hline & CAR & MP & Valor $t$ \\
\hline TABACO & & & Valor $p$ \\
Edad de inicio en el hábito de fumar & 13,3 & 11,7 & 4,912 \\
Promedio de cigarrillos consumidos semanales & 8,0 & 24,7 & $-3,386$ \\
ALCOHOL & & & 0,0001 \\
Edad de inicio en el hábito de fumar & 13,3 & 11,6 & 3,984 \\
Promedio de cigarrillos consumidos semanales & 9,3 & 29,1 & $-3,315$ \\
MARIHUANA & & & 0,0001 \\
Edad de inicio en el hábito de fumar & 13,5 & 11,6 & 2,846 \\
Promedio de cigarrillos consumidos semanales & 11,3 & 34,3 & $-3,003$ \\
\hline
\end{tabular}

$\mathrm{CAR}=$ cuestionario auto registrado, $\mathrm{MP}=$ metodología de pares.

del cuestionario auto registrado, y los consumidores de cocaína y pasta base, la cantidad promedio de pastillas de éxtasis que consumen es significativamente mayor con la metodología de pares que con la metodología del cuestionario auto registrado, como se observa en las Tablas 4 y 5 .

El consumo de drogas ilícitas fue de 17,7\% durante el año y de ellos, un 12,4\% también consumen actualmente, es decir, dos de cada tres escolares que declaran haber consumido durante el año, también lo hacen actualmente. Con la metodología de pares el consumo fue de 23,9\% durante el año y se registró que de ellos un 18,0\% consumen actualmente, cifras significativamente mayores a lo encontrado con el cuestionario auto registrado, que fue de $11,8 \%$ y $7,2 \%$ respectivamente, donde el consumo actual representa mayor porcentaje del consumo anual, con la metodología de pares (ver Tabla 6).

Al comparar el consumo anual por tipo de drogas entre ambas metodologías, se observa en la Tabla 7, que la 
Tabla 5: Promedio de pastillas de éxtasis, en consumidores anuales de cocaína y pasta base.

\begin{tabular}{|c|c|c|c|c|}
\hline TIPO DE CONSUMIDOR & CAR & MP & Valor $t$ & Valor $p$ \\
\hline \multicolumn{5}{|l|}{ COCAÍNA } \\
\hline Promedio de pastillas de éxtasis consumidos semanales & 1,50 & 8,00 & 7,506 & 0,042 \\
\hline \multicolumn{5}{|l|}{ PASTA BASE } \\
\hline Promedio de pastillas de éxtasis consumidos semanales & 1,50 & 8,00 & 7,506 & 0,042 \\
\hline
\end{tabular}

$\mathrm{CAR}=$ cuestionario auto registrado, $\mathrm{MP}=$ metodología de pares.

Tabla 6: Porcentaje de consumo de drogas ilícitas según metodología.

\begin{tabular}{llllccccc}
\hline Período de consumo & $\mathrm{n}$ & $\mathrm{CAR}$ & $\mathrm{n}$ & $\mathrm{MP}$ & $\mathrm{n}$ & Total & Valor $Z$ & Valor $p$ \\
\hline Último año & 18 & $11,8 \%$ & 34 & $23,9 \%$ & 52 & $17,7 \%$ & 2,717 & 0,005 \\
Últimos 30 días & 11 & $7,2 \%$ & 25 & $18,0 \%$ & 36 & $12,4 \%$ & 2,782 & 0,004 \\
\hline
\end{tabular}

$\mathrm{CAR}=$ cuestionario auto registrado, $\mathrm{MP}=$ metodología de pares.

Tabla 7: Porcentaje de consumo anual de drogas ilícitas según metodología.

\begin{tabular}{lcccccccc}
\hline Tipo de drogas consumidas último año & $\mathrm{n}$ & $\mathrm{CAR}$ & $\mathrm{n}$ & $\mathrm{MP}$ & $\mathrm{n}$ & Total & Valor $Z$ & Valor $p$ \\
\hline Marihuana & 17 & $11,2 \%$ & 30 & $20,4 \%$ & 47 & $15,7 \%$ & 2,153 & $0,021^{*}$ \\
Cocaína & 4 & $2,6 \%$ & 15 & $10,7 \%$ & 19 & $6,5 \%$ & 1,802 & $0,005^{*}$ \\
Pasta Base & 6 & $3,9 \%$ & 16 & $11,9 \%$ & 22 & $7,7 \%$ & 2,510 & $0,011^{*}$ \\
Éxtasis & 3 & $2,0 \%$ & 5 & $3,7 \%$ & 8 & $2,8 \%$ & $0,294^{* *}$ \\
Otro Inhalante & 4 & $2,6 \%$ & 3 & $2,0 \%$ & 7 & $2,4 \%$ & $0,511^{* *}$ \\
\hline
\end{tabular}

* prueba Z, ** prueba Fisher

$\mathrm{CAR}=$ cuestionario auto registrado, $\mathrm{MP}=$ metodología de pares.

Tabla 8: Porcentaje de consumo actual de drogas ilícitas según metodología.

\begin{tabular}{lcccccccc}
\hline Tipo de drogas consumidas último mes & $\mathrm{n}$ & $\mathrm{CAR}$ & $\mathrm{n}$ & $\mathrm{MP}$ & $\mathrm{n}$ & Total & Valor $Z$ & Valor $p$ \\
\hline Marihuana & 11 & $7,2 \%$ & 21 & $14,3 \%$ & 32 & $10,7 \%$ & 1,971 & $0,0244^{*}$ \\
Cocaína & 1 & $0,7 \%$ & 11 & $7,9 \%$ & 12 & $4,1 \%$ & 3,100 & $0,001^{*}$ \\
Pasta Base & 5 & $3,3 \%$ & 11 & $8,1 \%$ & 16 & $5,6 \%$ & 1,790 & $0,0367 *$ \\
Éxtasis & 2 & $1,3 \%$ & 2 & $1,5 \%$ & 4 & & $1,4 \%$ & $0,640 * *$ \\
Otro Inhalante & 0 & $0,0 \%$ & 2 & $1,3 \%$ & 2 & $0,7 \%$ & $0,244 * *$ \\
\hline
\end{tabular}

* prueba Z, ** prueba Fisher

$\mathrm{CAR}=$ cuestionario auto registrado, $\mathrm{MP}=$ metodología de pares.

declaración del consumo es significativamente mayor usando la metodología de pares en el consumo de marihuana, cocaína y pasta base, encontrando tasas de 20,4\%, 10,7\% y $11,9 \%$ respectivamente. Por otro lado, con el cuestionario auto registrado se declaró un $11,2 \%, 2,6 \%$ y un $3,9 \%$ $\mathrm{y}$ en el resto de las drogas no se encontraron diferencias significativas.

De acuerdo a lo encontrado respecto al tipo de droga, el consumo actual de marihuana, cocaína y pasta base es significativamente mayor utilizando la metodología de pares que con el cuestionario auto registrado. En los otros tipos de drogas no son significativas las diferencias declaradas, como se observa en la Tabla 8.

\section{Discusión}

En base a las evidencias encontradas en múltiples estudios, los grupos de jóvenes entre 18 y 25 años son los más vulnerables frente al consumo de drogas lícitas, lo que es una situación generalizada en el mundo. En cambio, el consumo de drogas ilícitas abarca un rango mayor de edad, situándose entre los 14 y 35 años. Por otro lado, hay estudios 


\section{Metodología de Pares y el Cuestionario Auto Registrado Estándar}

que muestran que el consumo de drogas ilícitas tiende a mantenerse en el tiempo en el género masculino y por otro lado, muestran el aumento en la cantidad del consumo, es decir, son los mismos consumidores pero que consumen mayor cantidad y/o más veces.

Destacamos que en nuestros datos no se detectó ninguna diferencia significativa en el consumo de drogas lícitas a nivel global, tanto en el consumo anual ni actual, como tampoco en el consumo específico de tabaco ni alcohol, lo que ratifica la hipótesis de que no habiendo situaciones de conflicto en la declaración, se puede usar indistintamente cualquier metodología.

Los resultados obtenidos permiten afirmar con algún grado de certeza, que existe sub declaración del consumo de drogas ilícitas por los escolares de género masculino, cuando se aplican métodos de recolección tradicionales.

Los resultados obtenidos en este estudio, sobre el consumo de drogas ilícitas utilizando la metodología del cuestionario auto registrado son, incluso, menores a los obtenidos en los estudios nacionales realizados en escolares, por el Consejo Nacional para el Control de Estupefacientes, año 2007; esto se debe a que la condición de consumo fue más estricta a la utilizada en este estudio. Por ejemplo el consumo anual de drogas ilícitas, en este estudio, se considera consumidor al que lo hace con una frecuencia de al menos una vez al mes, a diferencia de la definición que se usa en el Consejo Nacional para el Control de Estupefacientes, donde se considera consumidor al que lo hace al menos una vez al año.

Cabe destacar que los resultados encontrados son concordantes con los planteados por Wiebel (1990), donde hace notar el problema de la sub declaración en estos casos.

Los resultados del estudio ponen en evidencia la subdeclaración y por ende la sub estimación de la cantidad de jóvenes que consumen drogas ilícitas, lo que provoca preocupación y hace cada vez más necesaria una revisión de las cifras que se manejan, en especial en aquellas instituciones que tienen a cargo la prevención, tratamiento y curación de este mal.

Por otro lado, cabe destacar que se identificaron algunas limitaciones de la metodología de pares, por ejemplo en relación a que su aplicación está condicionada a poblaciones cerradas, que requiere de personal especializado para la selección de los entrevistadores y una supervisión del proceso de recolección. Para resolver estos problemas es que se considera fundamental generar factores de corrección para la prevalencia obtenida en los estudios a nivel nacional.

\section{Referencias}

Boland, M., Fitzpatrick, P., Scallan, E., Daly, L., Herity, B., Horgan, J. \& Bourke, G. (2002). Trends in medical student use of tobacco, alcohol and drugs in an Irish university, 1973-2002. Drug and Alcohol Dependence, 85,123 - 128.
Consejo Nacional para el Control de Estupefacientes [CONACE], (2005). Sexto estudio nacional de drogas en población general de Chile. Santiago: Gobierno de Chile.

Consejo Nacional para el Control de Estupefacientes [CONACE], (2006). Séptimo estudio nacional de drogas en población general de Chile. Santiago: Gobierno de Chile.

Consejo Nacional para el Control de Estupefacientes [CONACE], (2007). Séptimo estudio nacional de drogas en población general de Chile. Santiago: Gobierno de Chile.

Diagnostic and Statistical Manual of Mental Disorders [DSM-IV], (2005). Clasificación de Enfermedades Mentales de la Asociación Psiquiátrica de los Estados Unidos (APA). Washington: APA.

Encuesta Nacional de Adicciones (2002). Consultada el 15 de Marzo de 2009 en el sitio: http://www.conadic.gob.mx/interior/menu_superior/ investigacion_y_estadistica/enc_nal_adicciones_2002.html

Fountain, J. \& Griffiths, P. (1999). Synthesis of qualitative research on drug use in European Union: Report on an EMCDDA project. European Addiction Research, 5, 4-20.

Griffiths, P., Gossop, M., Powis, B. \& Strang, J. (1993). Reaching hidden populations of drug users by privileged access interviewers: Methodological and practical issues. Addiction, 88, 1617-1626. Article first published online: 24 JAN 2006, DOI: 10.1111/j.1360-0443.1993.tb02036.

Kohn, R., Levav, I., Caldas de Almeida, J. M., Vicente, B., Andrade, L., Caraveo-Anduaga, J. J., Saxena, S. \& Saraceno, B. (2005). Los trastornos mentales en América Latina y el Caribe: Asunto prioritario para la salud pública. Revista Panamericana de Salud Publica, 18, 229-40.

Kuebler, D. \& Hausser, D. (1997). The swiss hidden population study: Practical and methodological aspects of data collection by privileged access interviewers. Addiction, 92, 325-334. Article first published online: 24 JAN 2006, DOI: 10.1111/j.1360-0443.1997.tb03202.x

Medina-Mora, M. E., Cravioto, P., Villatoro, J., Fleiz, C., Galván-Castillo, F. \& Tapia-Conyer, R. (1998). Consumo de drogas entre adolescentes: Resultados de la Encuesta Nacional de Adicciones. Salud Pública Mexicana, 45, 16-25.

Organización Mundial de la Salud (2004). Neurociencia del consumo y dependencia de sustancias psicoactivas. Ginebra: OMS.

Pillon, S., O’Brien, B. \& Piedra, K. (2005). The relationship between drugs use and risk behaviours in brazilian university students. Revista Latino-americana de Enfermagem, 13, 1169-76.

Rodríguez, J., Fernández, A. M., Hernández, E. \& Ramírez, S. (2006). Conductas agresivas, consumo de drogas e intentos de suicidios en jóvenes universitarios. Terapia Psicológica, 24, 63-69.

Rodríguez, J., Fernández, A. M. \& Hernández, E. (2007). Descripción del consumo de drogas lícitas e ilícitas por género a través de la metodología de pares. Revista Médica de Chile, 135, 449-456.

Rodríguez, J. \& Hernández, E. (2005). Análisis de confiabilidad y validez de constructo de un instrumento que mide el consumo de drogas lícitas e ilícitas en estudiantes universitarios. Terapia Psicológica, 23, 83-90.

Rodríguez, J., Hernández, E. \& Cumsille, M.A. (2005). Implementación de la metodología de pares para estimar el consumo de drogas lícitas e ilícitas. Revista Chilena de Salud Pública, 9, 20-24.

Ruangkanchanasetr, S., Plitponkarnpim, A., Hetrakul, P. \& Kongsakon, R. (2005). Youth risk behavior survey: Bangkok, Thailand. Journal of Adolescent Health, 36, 227-235.

United Nations Office on Drug Control and Crime Prevention (2002). Global ilicit drug trends 2001. Nueva Cork (NY): UN ODCCP.

Villatoro-Velázquez J.A., Medina-Mora M.E., Hernández-Valdés M., FleizBautista C.M., Amador-Buenabad N.G. et al. La encuesta de estudiantes de Nivel Medio y Medio Superior de la Ciudad de México: noviembre 2003. Prevalencias y evolución del consumo de drogas. Salud Mental 2005,28(1):38-51.

Weiner, M., Sussman, S., Sun, P. \& Dent, C. (2005). Explaining the link between violence perpetration, victimization and drug use. Addictive Behaviors, 30, 1261-1266.

Wiebel, W. (1990). Identifying and gaining access to hidden populations. NIDA Research Monographs, 98, 4-11. 